\title{
Strategy Analysis of Listening Discourse Acquisition: Based on the Special Dictation Text Type
}

\author{
Fan Wang \\ School of Foreign Languages, Zhejiang Ocean University, Zhoushan, Zhejiang, 316022, China
}

\begin{abstract}
The dictation text has always been a weak point for our students in Test for English Majors-4. Discourse teaching is an indispensable part of listening teaching. This paper mainly discusses the pattern analysis and strategy methods in the four dictation text listening discourse. The content includes TEM-4 listening examination requirements, dictation text discourse analysis, the answer steps and strategy methods in dictation text. The research of this subject is designed to help students perform better in their listening exam.
\end{abstract}

Index Terms - strategy analysis, dictation text, test for English Majors-band 4, listening discourse

\section{INTRODUCTION}

TEM-4,Test for English Majors. Since 1991, it has been implemented by the Ministry of Education of the People's Republic of China to inspect students from all over the country. In the TEM-4 exam, the listening score is 30 points, of which dictation text has a large proportion. It puts great demands on students' comprehensive ability and individual skills. How to get a high score in the dictation text is a test of the learner's language skills.

\section{DictATION TEXT ANALYSIS}

The dictation part of TEM-4 exam is aimed at testing candidates' listening comprehension, spell proficiency, and the ability to use punctuation correctly. It not only examines students' language knowledge,but also tests candidates' psychological quality, sensory coordination and responsiveness. This section requires candidates to dictate a short essay about 80-90 words. The essays are widely selected and not illegitimate. The genre includes various forms such as narrative, description and explanation. The daily life and social campus are the main topics.The time of dictation text takes about 10 minutes, the score is 10 points.

\section{A. Theoretical Basis of Dictation Text Analysis}

In second language acquisition, discourse acquisition has higher learning efficiency than vocabulary and sentence acquisition, especially in listening activities. Traditional listening training only stays at the level of sentences, focusing only on the linguistic forms of pronunciation, vocabulary, grammar, etc., not the discourse analysis, which is inefficient. Some linguists apply discourse theory to English writing, reading and translation. However, the application of discourse theory to listening teaching is rare. Halliday believes that discourse analysis has two different levels of goals: 1 . To understand the meaning of the text itself, through language analysis to illustrate how the text expresses meaning and why there is such meaning; 2 . To analyze the cultural context and context of discourse, and also explore the relationship between discourse and context. Discourse usually refers to a linguistic whole composed of a series of consecutive segments or sentences, with logical rationality and formal coherence. Discourse analysis is mainly based on the third function (Metafunction) of the systemic functional linguist Halliday's functional grammar----the textual function. Discourse function refers to how people organize the information when they use language, and at the same time indicate the relationship between a piece of information and other, and also shows the relationship between the transmission of information and the communicative context in which the speaker is located. The discourse function has components such as the main structure, information structure, and coherence. Any discourse has a certain discourse structure, that is, the overall structure of the discourse, and the construct is composed of context variables associated with the actual context. The same type of discourse structure has roughly the same context variable. Halliday and Hasan summarized these relatively fixed discourse structures into the discourse structure potential, referred to as SP., which includes at least four aspects: 1.Essential elements, which are essential for any type of discourse. 2. Alternative elements; 3 . the order in which the essential and optional elements appear.4. Recursion potential, that is, whether a change in an element will necessarily cause another one. Understanding discourse in the practice of listening, especially the structural characteristics of short texts can improve the level of listening comprehension.

\section{B. Features of Dictation Discourse in Tem-4}

As stated in the Tao Te Ching: "The knower does not speak, the speaker does not know." In other words, "listening" 
rather than "speaking" is the root of learning and the source of wisdom. The dictation part of the Professional Level 4 exam is designed to test candidates' listening comprehension, spell proficiency, and the ability to use punctuation correctly. It not only examines students' language knowledge and professional skills, but also tests candidates' psychological quality, sensory coordination and responsiveness. (Zou Ke, 2010)The essays are widely selected and not illegitimate. The genre includes various forms such as narrative, description and explanation. The daily life and social campus are the main topics. Moderate. The dictation part takes about 10 points, out of 10 points. The dictation recordings are recorded by experts from the United Kingdom and the United States, with English or American sounds, without any local accent. The dictation part has a wide range of subjects and diverse genres. The following topics are from the dictation part of the Professional Level 4 exam (2009-2018):

2009 New Year's Eve holiday

2010 Freshmen's Week society

2011 British Holidaying Habits holiday

2012 Eco-tourism tourism

2013 What Are Dreams for? dream

2014 Limiting the Growth of Technology technology

2015 Male and Female Roles in Marriage culture

2016 Think Positive and Feel Positive learn

2017 Learning Sympathy learn

2018 Emotional Reaction to Music learn

Discourse structure of all these topics are similar in many linguistic aspects, such as vocabulary, sentence and grammar. Take 2014 audio-script as an example:

Limiting the Growth of Technology

Throughout history man has changed his physical environment to improve his way of life./With the tools of technology man has altered many physical features of the earth./He has transformed woodland into farmland./He has modified the face of the earth by cutting through mountains to build roads and railways./However, these changes in the physical environment have not always had beneficial results./Today, pollution of the air and water is a danger to the health of the planet. Each day thousands of tons of gases come out of vehicles./Smoke from factories pollutes the air of industrialized areas and the surrounding countryside./The air in cities is becoming increasingly unhealthy./The pollution of water is equally harmful./In the sea pollution from oil is killing a lot of sea plants and fish./It is now necessary for man to limit the growth of technology in order to survive on earth.

Article analysis: Among them, the long sentence has a maximum of 15 words, and the short sentence has a shortest length of only 7 words. The content is introduced the impact of technological development on life. The context is clear, the difficulty is intermediate, there is no singular words and complex background knowledge, and it can be used as a special four-level training course.

In the course of listening and writing, we should obey the rule of language learning. The background knowledge in the process of listening comprehension in short texts is especially important. It can make the listener predict the next content of the discourse based on the overall characteristics of the discourse. On the one hand, it can reduce the difficulty of understanding of subsequent listening, on the other hand, it can control the force of injection, suppress irrelevant associations appearing in the brain, and avoid the phenomenon of distraction. Based on the above characteristics, we can find the rules of language use in short texts in the practice of listening teaching, and find out the teaching methods that can help improve listening comprehension through analysis. (Fang Weiwen, 2008)

\section{The Strategy Analysis of Dictation TeXT In TEM-4}

Listening strategy, as an crucial part of language learning strategy, with learning strategy as the theoretical framework, (Fang Weiwen, 2008)can be understood as deliberate behaviors or psychological movements of the learner in order to improve the listening level when dealing with the information they hear. On the classification of listening strategies, scholars at home and abroad express their opinions. Among them, the generally accepted classification method is Learning Strategy Trilogy by O'Malley \& Chamot. According to them, Learning strategy refers to the "way of input" of the learner, i.e., how to process, store, and retrieve what they learn in the brain. (Ellis, 1985) In this sense, learning strategy is learning methods, management ways and various activities to employ language during the process of learning. Appropriate learning strategies will help L2 learners improve their TL competence while inappropriate ways will directly lead to fossilization. The three classification of cognitive strategies, metacognitive strategies and social affective strategies is followed by foreign scholar Vandergrift (1997). Based on the specific description and classification, he constructed a relatively complete listening strategy.

\section{A. Answer Steps in Mini-lecture}

During the exam, the dictation materials were totally read four times. The first time is read at normal speed, and the speech rate is 120 words per minute, so that the candidates can understand the material. When the second and third readings are made, leaving a gap of about 15 seconds between the groups, so that the candidates can write. The fourth time, then read at normal speed, let the candidates check. After the dictation recording is finished, the candidate has two 
minutes of review time. So it ask us to grab the key points and make a response in the fastest time. To achieve this, the following guidelines must be observed.

1. To understand the general idea for the first time

After understanding the topic, you need to immediately grasp the time before the start of the essay text for fast prediction. The prediction should be targeted, mainly by using your own background knowledge and writing experience, association with the topic, possible vocabulary into a phrase; association and essay topic related synonyms, such as $(08$ years paper) career; similar and appear in the text words have job, work, occupation, position, etc.; associations and essays related to the tortuous change words, such as (07 years)advertising can be associated with the advertises, advertisement; association and essay topic related to the relationship between the upper and lower meaning, such as (06 years) the Internet has a relationship of ups and downs and the words appearing in the text are online, games, chat rooms, etc. The first time you listen to the sound, you will comprehensively grasp and integrate the meaning of the text. The key point is to understand the general idea: if you can't understand all of them, you can also integrate the cultural camp by catching the key essays in the text. For example, when listening to the 2009 New Year's Eve, according to the friends or family parties, drinks, fireworks, clock, 12, resolution in the text, you can reason that the essays are connected in tandem, that is, on New Year's Eve, people will reunite with friends and family. Have a party, or go out for a drink. Fireworks will be released that night. At 12 o'clock in the evening, people will count down to meet the New Year and make a New Year's wish...

2. To write the second and third times while listening

Pay attention to the first sentence and the outline. The first sentence or the first paragraph of the essay is often a summary of the content of the essay, such as the purpose of the talk, the main content, the author, the argument, the time, place and cause of the story. Therefore, if you grasp the first sentence during dictation, you can usually catch the essay. These major information can contribute to then grasp the background knowledge of the essay is conducive to dictation. Grasp the group and capture keywords. When reading the recording in the second time, you must first understand it in units of meaning group, you should grasp the sentence structure and keywords to record, and then try to make up other words when listening to the third time.

Important information keywords include: different forms of verbs, nouns indicating time, place, characters, adjectives and figures indicating the person's emotional attitudes.

3. Leaning filling for the fourth time

The fourth time is read as normal speech rate, and there are two minutes of review time. Candidates should seize the time to check carefully and correct some mistakes caused by carelessness and neglect.

\section{B. Learning the Strategy Methods in Dictation Text}

Confucius said: If a worker wants to do something good, he must first sharpen his weapon. How to build confidence in the exam? We also need to master certain strategic methods in listening learning. There are some methods which can help you overcome the difficulties better in listening.

1. To overcome anxiety and build self-confidence

It is a natural phenomenon that words, sentences and even some contents cannot be understood in the dictation text. Fang Wenwei said that the principle of redundancy of discourse information, this part of the information which not be understand may be reappeared in other forms elsewhere in the discourse, or it can be reasonably guessed by the context or background knowledge of the discourse. On the basis of the potential structure SP of the discourse, the content of the necessary components of the discourse can be inferred, and this part may be only an auxiliary element, even if it is not understood, it may not affect the listening. In this way, students can truly establish a psychological advantage and lay a good foundation for rational speculation and prediction using textual features. (Fang Weiwen, 2008)

2. To strengthen the basic skills

Improve the English listening level, familiar with the different ways of listening and pronunciation of English and American families, and the pronunciation and intonation. Einstein said that "interest is the best teacher." It is conceivable that learning interest plays an important role in our life. In our free time, we can watch some of the classic English subtitle movies that we are interested in, and consciously imitate and follow, extract some authentic phrases, classic famous phrases and interesting background knowledge. Sometimes it is a background error, let us lose points in the exam. For example, ridiculous mistakes can occur because of the misunderstanding of the sound, but without the use of background knowledge or common sense to judge the meaning of the words or sentences that are heard. For example, due to the similar pronunciation of civil and silver, candidates have mistakenly listened to civil as silver. But according to background knowledge, we know that in the United States, marriage can be held in a religious ceremony or in a civil ceremony. Therefore, the civilization is correct.

The dictation text discourse as a model for learning English phonetics is also an important medium for us to learn grammar and vocabulary. While watching American TV dramas and listening to the radio, we expanded our vocabulary and knowledge likewise. As an English major, we must be proficient in the words and idioms listed in the syllabus. Constantly improve your global vision, and get more exposure to new words and new knowledge. In the meantime, the old words can not be neglected, so that you can learn new things and use more words. In addition, you should collect and sort out some words with multiple meanings. In this way, even if you encounter strange words or use the familiar words newly, you can be not afraid of making mistakes. Of course, a large number of intensive readings, extensive 
listening, and long-term reciting of some world famous articles should be conducted.

Professional level four not only examine our expertise, but also our extracurricular knowledge. The mini-lecture listening discourse is a model for learning speech and an important medium for us to learn grammar and vocabulary. While watching the American TV dramas and listening to the radio, we have also expanded our vocabulary and knowledge. The standard vocabulary of the English Major CET is 8,000 words, and you need to master at least 5,000 words. As an English major, we must be proficient in the words or idioms listed in the syllabus. Constantly improve your global vision, and get more exposure to new words and new knowledge. At the same time, the old words can not be neglected, so that you can learn new things and use more words. In addition, you should collect and sort out some words with multiple meanings. In this way, even if you encounter strange words and use the familiar words flexibly, you can not be afraid and courageous.

3. To be careful with the listening traps

Misunderstanding is one of the most common mistakes in our dictation text. The difference between English pronunciation and American pronunciation, the distinction between homonyms and near-sound words, the mastery of weak-sounding syllables, incomplete plosives, and even pronunciation. In consequence, when listening to an article, be sure to judge which word is based on context, etc. There are the following common mistakes:

The difference between English pronunciation and American pronunciation, the resolution of homophones and nearsound words, weak syllables, incomplete plosive, continuous pronunciation .Also, writing mistakes is also a mistake we have made. Such as capitalization problem, grammatical error, background knowledge error and punctuation error.

4. "Listening" and "speaking" combination

In the current classroom, there are often "listening" and "speaking" separations, with separate listening and speaking classes. In fact, They are interdependent and interact with each other affects. In line with the theory of communication, the core of the process of verbal communication is the transformation between the linguistic sign and the meaning it represents. The speaker must translate meaning into sound (linguistic symbol), and the listener should transform the sound into meaning. This transformation process is mainly done by the brain, the vocal organs and the listening organs. Therefore, to complete the conversion activities, listening and speaking not only rely on the common physical, physiological and psychological foundations, but also in the process of conversion, the two influence each other and promote each other, the basis of listening is the basis, providing a true context for the theory. It is the precondition for listening, and the quality of listening can be tested to promote the mastery of listening skills. Therefore, in order to develop students' oral communication ability in the teaching of English, it is necessary to combine listening and speaking to cultivate, in light of absenting language environment, we need to create a real context of the classroom, so that students have an opportunity both listening practice and speaking. Gradually, it makes listening and speaking skills develop together and improve the level of oral communication. (Gao Mingxia, 2001)

We can use copious practical teaching methods to let students speak out in the listening class. For example, after listening to the recording or watching the video materials, students should repeat as much as possible in English according to the notes taken. If there is difficulty, the teacher can be given some questions as a guide. (Yang Yan,2014) To encourage students to open their mouths as much as possible. Only when they can speak can they listen, only when they can listen can they write correctly. This relates directly to the question I put forward before.

5. To develop shorthand and predictive ability

Shorthand is a basic skill that students need to master when doing physical problems. (Luo Xuelin, 2016) On one hand, it can reduce the difficulty of understanding of subsequent listening, on the other hand, it can control the force of injection, suppress irrelevant associations appearing in the brain, and avoid the phenomenon of distraction. Sometimes, in the dictation text listening test, we clearly understand each word, but we can't write all the content. Due to the distinction in students' memory, the meaning of more than ten words and sometimes even eight words can only be recorded in the first half or half of the sentence. Seriously affecting the integrity of the group, it also makes students lose confidence. In addition to deliberately practicing memory, students should be trained to develop good dictation and writing habits. That is, while listening and writing, first write the meaning of the group structure. We can start writing immediately at the beginning of the second reading. You don't need to understand the meaning of the words. First, write down the first letter or highlight the consonant. When writing, use the notional word and the group of the subject as the object structure, back to supplement after. This can use the pen to make up for the weakness of memory.

At the same time, when we practice after class, we must also consciously use the group of meaning to understand and remember. If time is too late, you can use your familiar symbol abbreviations instead. Use the dictation method to select the appropriate writing skills. Listening and writing are a pair of spears and shields, so the relationship between the two must be handled correctly. In dictation, writing should be based on the following three steps: 1 . listen; 2 while listening and writing; 3 listening while checking.

The first time is the full text reading, should pay attention to the overall understanding of the text, (Wang Ping, Liu Hongquan, 2008)properly fill in some words and make some notes, to listen to the main, as a supplement; the second and third times are repeated in sentences, to If the group is paused, the candidate can use the pause between the sentences to record the content, and should do both dictation and listening while listening. At the last time, check and check the main points of the content, and minimize the grammar, spelling, etc. in the language error.

6. To learn to choose according to the scoring method 
After the dictation part of the scoring standard reform, each of the ten essays scored independently. Each group is mainly based on the size of the dictation error. Including singular and plural, capitalization, definite article and other levels of deduction. In the dictation material, the long-term group accounts for $30 \%$, the medium-length group accounts for $40 \%$, and the short-term group accounts for $30 \%$. Two big mistakes in one group of meanings are zero points.

Therefore, we must distinguish between key points and non-key points when listening. We must strive to score ,meanwhile, we should learn to choose in accordance with the scoring standards.

\section{CONCLUSION}

The English National Level 4 Unified Test Listening Test is a composite part. The level of dictation depends on the ability to listen and write. The key to improving dictation is to strengthen all kinds of relevant knowledge, improve the training of skills, master the language structure, the overall understanding of the text and the memory. This paper is designed to analyze the features of dictation text in TEM-4 and relevant strategies to improve language proficiency. In order to further take advantage of the dictation form, more empirical researches are needed and intensive study should be conducted. Although this study is important for L2 learners, there are many deficiencies in it. For this reason, it is actually not convincing and persuasive. It is hoped that the strategy analysis of dictation text be conducive to the improvement of learners' performance. The conscious training of listening strategies is beneficial for candidates to improve their self-confidence and promote the coordinated development of cognition, emotion, skills and interest, will other aspects of health development. To some degree, it will help students improve the students' listening level and have a better language applied ability.

\section{ACKNOWLEDGEMENTS}

The paper was supervised by Miss Ma Lihua, who is an associate professor in the school of foreign languages, Zhejiang Ocean University, Zhejiang, China

\section{REFERENCES}

[1] Ellis, R. (1997). Second Language Acquisition. Oxford University Press.

[2] Fang Weiwen. (2008). Discourse Analysis and News Listening Teaching, Journal of Hunan Institute of Science and Technology, 29,172-174.

[3] Gao Mingxia. (2011). On the inner connection and speaking of listening and speaking. Yanbian university master's thesis.

[4] Luo Xuelin, (2016).Discussion on Teaching Methods of English Listening Tests, Modern Business and Trade Industry, $29,172$.

[5] Liu Shaolong, Wang Liuqi, Fu Bei, Ye Changqing \& Wu Zhiwei et al.(2017). Professional Level 4.GZ:World Book,1-4

[6] Selinker, L. (2013). Second Language Acquisition: An Introductory Course. Routledge.

[7] Tang Jing. (2005). English Majors Level 4 Difficulties in Dialysis (1)—Dictation, Exam, New Oriental English (University), 5, 6-11.

[8] Wang Ping, Liu Hongquan, (2018). English four-level listening skills, Foreign language teaching and research, 10,110.

[9] Yang Yan. (2014). On the misunderstandings of the 4th and 8th grade listening preparations for English majors, Foreign Language Education and Translation Development Innovation, 3, 77-79.

[10] Zou Ke. (2010). Preparing for the Four Listening from Various Aspects, Contemporary Education Forum, 4, 87-88.

Fan Wang was born in Gansu, China in 1999. She is a sophomore student. She is currently a student in the school of foreign Languages, Zhejiang Ocean University, Zhejiang, China. 